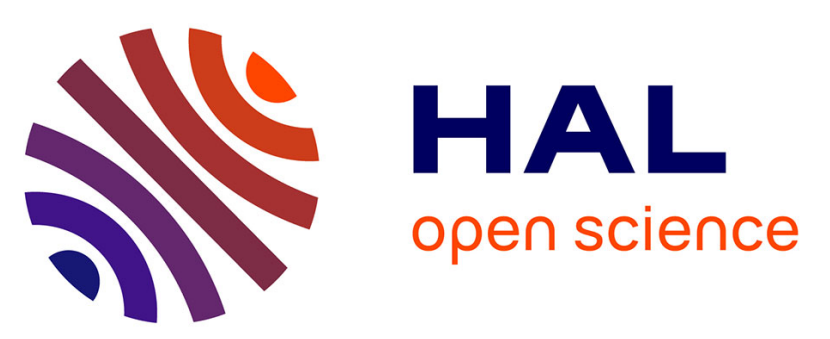

\title{
Functional Connectivity Driven by External Stimuli in a Network of Hierarchically Organized Neural Modules
}

\author{
Vladyslav Shaposhnyk, Pierre Dutoit, Stephen Perrig, Alessandro E. Villa
}

\section{To cite this version:}

Vladyslav Shaposhnyk, Pierre Dutoit, Stephen Perrig, Alessandro E. Villa. Functional Connectivity Driven by External Stimuli in a Network of Hierarchically Organized Neural Modules. 20th International Conference on Artificial Neural Networks, Sep 2010, Thessaloniki, Greece. pp 135-144, 10.1007/978-3-642-15819-3_18. inserm-00624112

\section{HAL Id: inserm-00624112 https://www.hal.inserm.fr/inserm-00624112}

Submitted on 15 Sep 2011

HAL is a multi-disciplinary open access archive for the deposit and dissemination of scientific research documents, whether they are published or not. The documents may come from teaching and research institutions in France or abroad, or from public or private research centers.
L'archive ouverte pluridisciplinaire HAL, est destinée au dépôt et à la diffusion de documents scientifiques de niveau recherche, publiés ou non, émanant des établissements d'enseignement et de recherche français ou étrangers, des laboratoires publics ou privés. 


\title{
Functional connectivity driven by external stimuli in a network of hierarchically organized neural modules
}

\author{
Vladyslav Shaposhnyk ${ }^{1,2}$, Pierre Dutoit ${ }^{1,3}$, Stephen Perrig ${ }^{3}$, and \\ Alessandro E. P. Villa ${ }^{1,3,4}$ \\ 1 Neuroheuristic Research Group, Grenoble Institute of Neuroscience, \\ Université Joseph Fourier, Grenoble, France \\ 2 Non-linear Analysis Department, Institute for Applied System Analysis, \\ State Techical University "Kyivskyy Politechnichnyy Instytut", Kiev, Ukraine \\ ${ }^{3}$ Sleep Research Laboratory, Dept. of Psychiatry Belle-Idée, Hôpitaux Universitaires \\ de Genève, Switzerland \\ 4 Neuroheuristic Research Group, Information Science Institute, \\ University of Lausanne, Switzerland \\ http://www . neuroheuristic.org/
}

\begin{abstract}
Complex neural modules with embedded neural development and synaptic plasticity features have been connected to form a hierarchical recurrent circuit. Virtual electrodes have been used to record a "neural" generated signal, called electrochipogram EChG, from each module. The EChG are processed by frequency domain methods to determine the modifications in functional connectivity by assessing quadratic phase coupling. The experimental paradigm is aimed to describe what happened prior to, at the beginning, towards the end, and after repeating an external input at fixed frequency. The results are discussed by comparing with the same signal processing methods applied to a human study.
\end{abstract}

Key words: spiking neural networks, hierarchical neural networks, distributed computing, computational neuroscience, EEG

\section{Introduction}

At mesoscopic level, the recording of brain activity by means of electroencephalography (EEG), electrocorticography (ECoG) and local field potentials (LFP) collects the signals generated by multiple cell assemblies. The neurophysiological processes underlying those signals are determined by highly non-linear dynamical systems [1]. Because of these nonlinearities the functional interactions between brain areas that are simultaneously sampled by electrophysiological techniques generate signals that can be better analyzed by third order polyspectral methods that retain phase relationships [2]. This analysis was applied to EEG by pioneers as early as the 1970s [3]. Phase coupling frequencies can be 
interpreted as frequencies of resonance of standing waves whose wavelength is associated to the average distance between interacting cell assemblies $[4,5]$.

In the present study we simulate the activity of interconnected neural networks undergoing neural developmental phases. The implementation of such complex models requires high performance of the simulation that can be achieved thanks to a powerful hardware platform, its bio-inspired capabilities, its dynamical topology, and generic flexibility of artificial neuronal models presented elsewhere $[6,7]$. The outcome is the implementation of each neural network into a Ubidule and a network of Ubidules as a Ubinet. Within each Ubidule the emergence of functional connectivity driven by neural development, cell and synaptic pruning, and selective external stimuli was assessed by recording Electrochipograms (EChG) which are analog signals similar to EEG generated by virtual electrodes located into each Ubidule [8].

The experimental paradigm is aimed to describe what happened prior to, at the beginning, towards the end, and after repeating an external input at fixed frequency. The rationale is that the spike timing dependent plasticity (STDP) embedded in the neural network models would drive the build-up of auto-associative network links, within each Ubidule, such to generate an areal activity, detected by EChG, that would reflect the changes in the corresponding functional connectivity within and between Ubidules. This experiment is compared to a small set of recordings performed in patients suffering of primary insomnia whose EEG recordings were analyzed during several sleep phases, before and after a clinical treatment.

\section{Hybrid system implementation}

The Ubidule is a custom reconfigurable electronic device allowing an implementation of several bio-inspired mechanisms such as growth, learning, and neural processing [9]. The common Ubidule platform is an hybrid system with an XScale-class processor that manages the software components of the system, such as ontogenetic processes, communications with other Ubidules, monitoring and recording of the activity. This processor is equipped with an open hardware subsystem which allows connecting any sort of USB device (sensors, actuators, Wifi / Bluetooth dongles, mass storage, etc.). The processor runs an embedded Linux operating system which facilitates Ubidule programming and management while ensuring portability at the same time.

Both hardware and software platforms are based upon modular architecture that offers interoperability among the hardware and the software parts of the system and simplifies the usage of bio-inspired features of the hardware. The neural system simulator consists of multiple computational modules, each one corresponding to a neural network, exchanging their neural activity and/or receiving input data from hardware sensors (camera, photodiode, radars, etc.) and/or providing output to hardware actuators (motor, diode array, etc.). The characteristics of the implementation naturally geared the modeling framework towards agent oriented programming. An evaluation of the available platforms 
of this kind led us to select JADE [10] for the development and runtime execution of peer-to-peer applications which are based on the agent oriented paradigm [11]. It is a JAVA-based multi-agent development system that fulfils the FIPA specifications [12].

In this study each network is a $2 \mathrm{D}$ lattice of $20 \times 20$ units that includes $80 \%$ of excitatory units and $20 \%$ of inhibitory units. Our framework implements several features of brain maturation, including apoptosis active during the very initial 700 time units and STDP active from the end of apoptosis until the end of simulation. This framework was extensively described elsewhere $[13,6,7]$. Synaptic pruning occured when the activation level of a synapse reached a value of zero, so that besides cell death and axonal pruning of dead cells provoked by apoptosis, the units whose all synaptic connections were characterized by a zero level of activation were definitely eliminated from the network. All units were simulated by leaky integrate-and-fire neuromimes with background activity used to simulate the effect of afferences that were not explicitly simulated within a network. The background activity to each neuron was set to 900 spikes/s with a low amplitude $(1 \mathrm{mV})$ generated by uncorrelated Poisson distributed inputs. In each Ubidule two sets of 20 excitatory units were randomly selected among the excitatory units corresponding to the "input" and "output" layers of the Ubidule. The neurons of these layers send and receive connections from the other units of both types (excitatory and inhibitory) within the network in addition to the connections with other Ubidules.

Our circuit topology remained fixed during all simulations and the Ubidules were characterized by their role in the network, i.e., sensory, processing, or motor (Fig. 1). In our network, the u1Sensory Ubidule has a pure sensory role. Ubidules labeled u3Process, u4Process, u5Process, u6Process have a pure information processing role and are characterized by having neither external inputs nor afferences from the motor Ubidule. They are all reciprocally interconnected and send efferent projections to u2Motor.

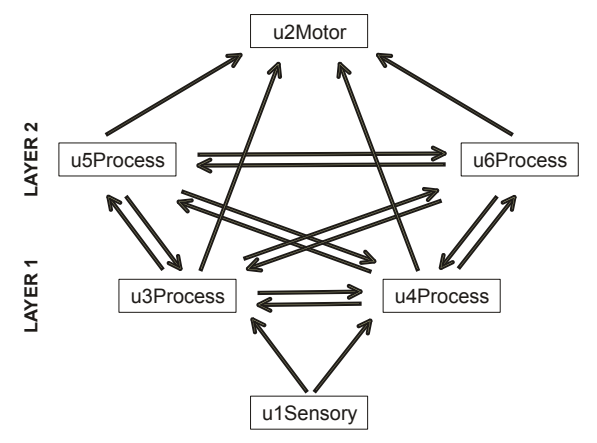

Fig. 1. The Ubinet hierarchical circuit used in all simulations. Solid arrows depict connections and directions of information flow between the Ubidules. 


\section{$3 \quad$ Electrochipograms}

Our design of the bio-inspired artificial neural networks allowed us to implement realistic virtual electrodes to record neuro-mimetic signals, called Electrochipograms (EChG), characterized by dynamics and features similar to those recorded in living brain structures. In our implementation the virtual electrode measures the potentials over a certain 'area' of the 2D lattice neuronal network according to an appropriate weighted sum [8]. The main parameters of the electode are its position over the neural network and its sensibility function. The tip of the virtual electrode was located in the middle of the 2D lattice of each Ubidule neural network. The sensibility function depends only on the distance between a given point of the lattice and the centre of the electrode field. According to this model, all neurons located at the same radial distance from the center of the electrode field make an equivalent contribution to the final electrode output and thus form an equi-potential layer [8]. In this study, the sensibility radius was set equal to 9 with a linear decaying function.

The EChG was recorded with a 6 channels virtual electrode system with one channel per Ubidule during 350 trials. Each trial had a fixed duration and included two intervals: a stimulation interval followed by an inter-stimulus interval. The stimulation was generated by spatio-temporal external stimuli applied only to the input layer of u1Sensory lasting 128 (Type A) and 512 (Type B) time steps. The group of simulations with higher stimulation frequency $(0.89 \mathrm{~Hz})$ was called "Simulations A" and the group with lower stimulation frequency $(0.67 \mathrm{~Hz})$ was called "Simulations B". The extensive use of Fast Fourier Transform in our signal analysis imposed, for improved efficiency, sampling frequencies which are powers of two. In practice the time-steps of the simulator were selected for convenient time units, i.e., 1024 time steps corresponding to $1000 \mathrm{~ms}$. The inter-stimulus interval was always equal to $1000 \mathrm{~ms}$. The recording time was divided into four periods defined following the amount of time the Ubinet was exposed to the stimulation: (i) PRE-learning beginning at time zero and lasting 27 trials characterized by the absence of any external stimulation (i.e., only the background activity was present during the stimulation interval); (ii) EARLY-learning lasting 50 trials, between trials \#28 and \#77; (iii) LATE-learning lasting 50 trials, between trials \#228 and \#277; and (iv) POST-learning lasting 50 trials, between trials \#278 and \#327 again characterized by the absence of any external stimulation.

The signals recorded during the stimulation interval were averaged across several trials in order to compute evoked potentials (e.g., Fig. 2). The signals recorded during the inter-stimulus interval were used for frequency domain analyses that included power spectrum, bispectrum and bicoherence analyses.

\section{Power Spectrum Analysis}

Figure 3 shows the averaged evoked potentials for the "first" $(u 3 P, u 4 P)$ and the "second" ( $45 P, u 6 P)$ processing layers and their corresponding Power Spectrum 


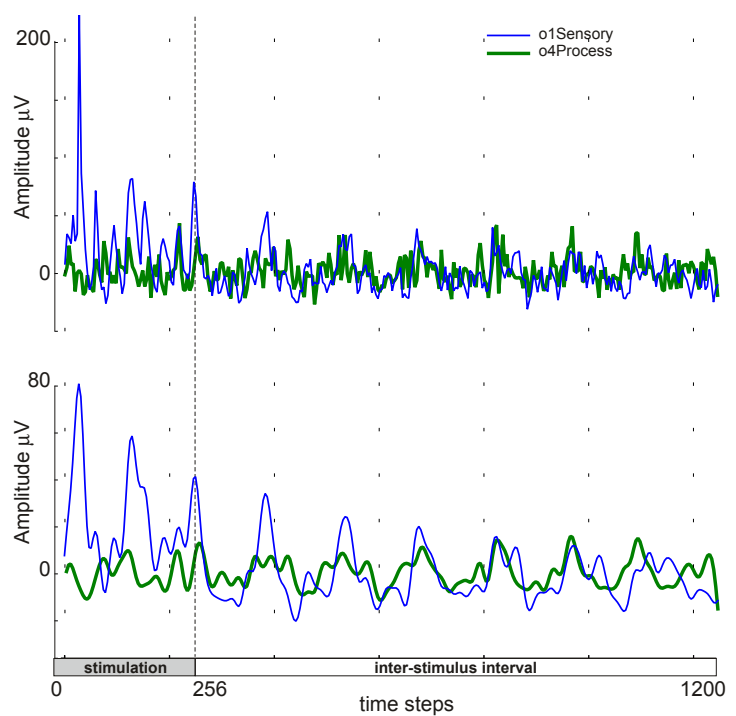

Fig. 2. Evoked potentials averaged over 50 trials obtained from u1Sensory (blue solid trace) and from u4Process (green dotted trace) Ubidules during the EARLY-learning stage. The stimulus was applied during 256 time steps. The upper panel displays the raw evoked potentials and the lower panel shows the signals smoothed by a Blackmann smoothing window in order to emphasize the low frequency components.

Densities (PSD). In the PSD several peaks could be observed around $10 \mathrm{~Hz}$, $15 \mathrm{~Hz}$ and $25 \mathrm{~Hz}$. The results obtained during the EARLY-learning stage were not significantly different from the $P R E$ recording condition. This suggests that PSD is little affected by the stimulus structure and by the subsequent functional connectivity at the begin of the stimulation. This is probably due to the fact that stimulus-driven selective cell and synaptic pruning were not yet producing any effect. During the LATE period the PSDs were characterized by a generalized decrease in the power and the preservation of the peak near $10 \mathrm{~Hz}$ with a noticeable decrease of the other peaks. It is interesting to notice that in the POST-learning stage the multiple peaks tended to appear again, thus suggesting that they are mainly driven by the combined effect of background activity and internal features of the model. Another general observation is that in mature networks, i.e. during the LATE- and POST-learning phases in comparison with $E A R L Y$ - and PRE-learning phases, PSD is getting lower, which means the total amount of energy transferred by the neural networks is decreasing. The POST-learning phase was characterized by $3.5 \mathrm{~dB} / \mathrm{Hz}$ lower values of power than appropriate values during PRE-learning phases. This decrease is likely to be associated to the pruning of synpatic links and cell death. 

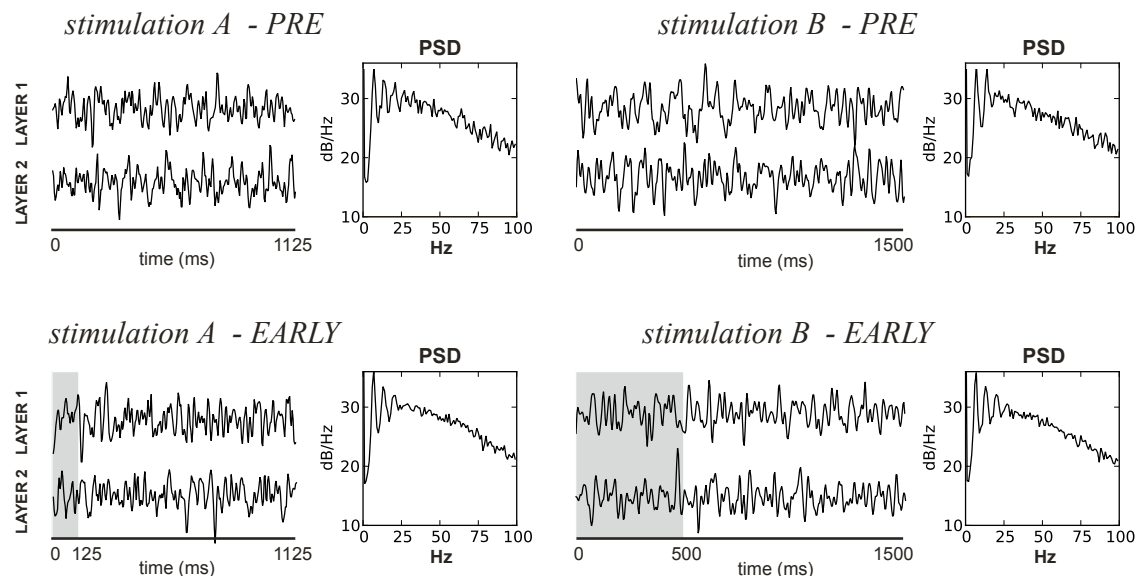

stimulation A - LATE
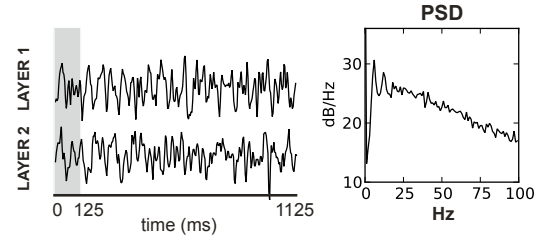

stimulation $B$ - LATE
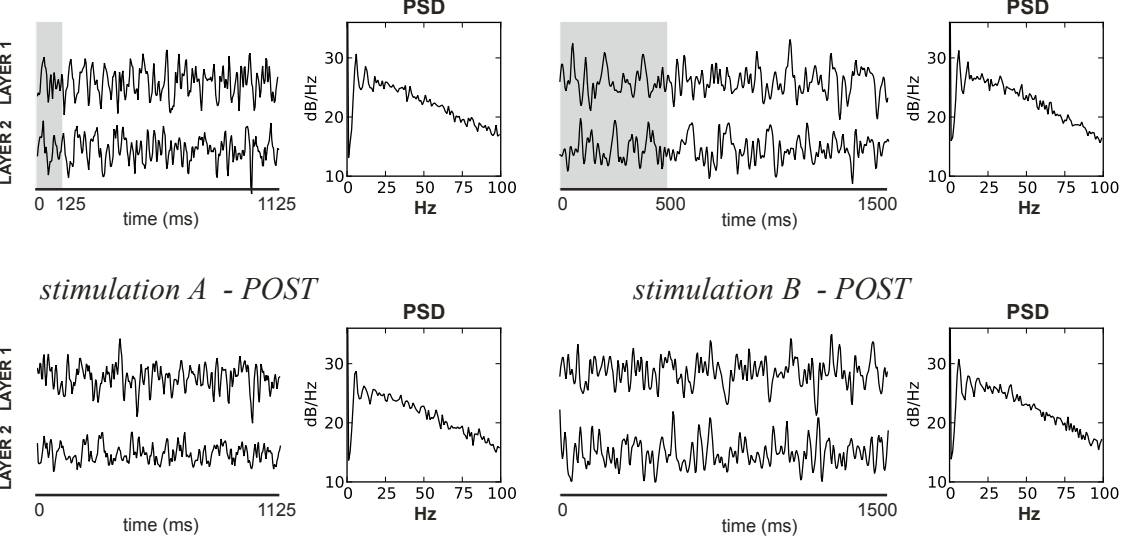

Fig. 3. Evoked Potentials and Power Spectrum Densities for the averaged recordings of the pair of Ubidules in Layer 1 and in Layer 2. The left panels correspond to stimulus Type A and the right panels to stimulus Type B. The gray stripes correspond to the periods of stimulation. From top to bottom the results referred to the PRE-learning, EARLY-learning, LATE-learning and POST-learning periods.

\section{$5 \quad$ Quadratic Phase Coupling}

The bispectral analysis was performed for all channels separatedly and the values of phase-coupled frequencies (i.e., the frequencies of resonance $f_{3}$ ) were determined. Let us consider the distribution of all phase-coupled frequencies $f_{3}$ observed in single-channel and cross-channel analyses. Let us consider the frequency band $] 1-24] H z$ for EChG and $L F$ the relative number of $f_{3}$ falling into this low frequency range. Let us consider the frequency band $] 60-84] \mathrm{Hz}$ and $H F$ the relative number of $f_{3}$ falling into this high frequency range. The index of resonant frequencies IRF is defined in the range 0-100 as follows: 


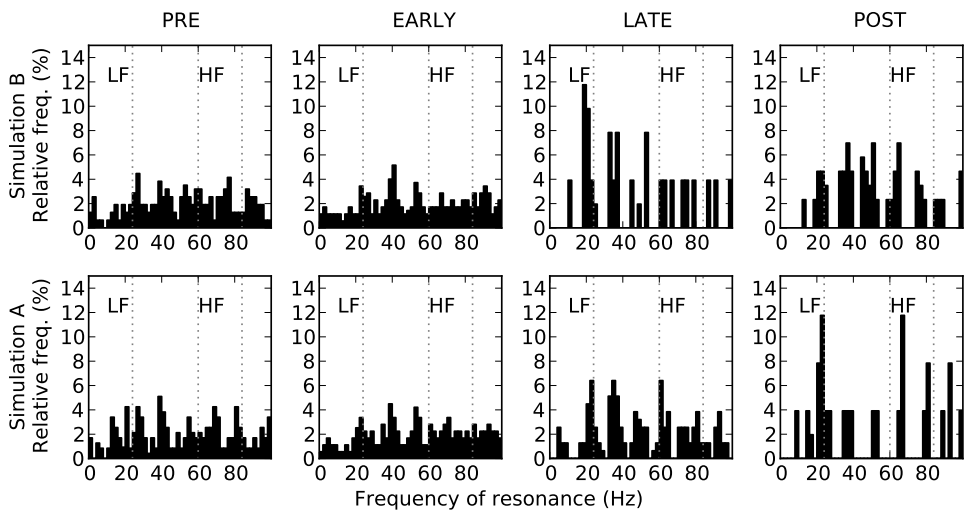

Fig. 4. Relative distribution of the frequencies of resonance for each period for Simulations $\mathrm{A}$ and $\mathrm{B}$. Bin size corresponds to $2 \mathrm{~Hz}$ intervals. The dotted lines delineate the limits of $L F$ and $H F$ bands.

$I R F=\frac{1}{2} \times\left(100+\left(\frac{H F-L F}{H F+L F} \times 100\right)\right)$. A value of $I R F$ close to 100 corresponds to a shift of $f_{3}$ towards higher frequencies and value of $I R F$ close to 0 corresponds to a shift of $f_{3}$ towards lower frequencies. $I R F$ values close to 50 indicates the phase-coupling was equally distributed in low- and high-frequency bands. The raw frequency ratio is simply defined by $R F R=\frac{L F}{H F}$. This means a large value of $R F R$ corresponds to a shift of phase-coupling towards higher frequencies and a low value of $R F R$ corresponds to a shift towards lower frequencies.

Figure 4 shows the distribution of $f_{3}$ in the range 1 to $100 \mathrm{~Hz}$ during all recording periods and for the two types of stimulus used in the Ubinet simulation. These histograms show a shift towards an increase in low-frequencies resonances during the LATE-learning phase, especially when compared with the distribution during the POST-learning, when the input stimulus was absent. The quantitative assessment of this analysis presented in Table 1 emphasizes the change in the value of $I R F$ between $E A R L Y$ - and LATE-learning phases. IRF $\approx 60$ decreased to $I R F \approx 14$ followed by an increase to the range 26-29 during the POSTlearning phase suggests that the shift towards low frequencies of phase-coupling was provoked by the learning protocol and not only due to the maturation of the network. The analysis of IRF and RFR shows also that in the POST-learning stage the resonant features remained affected by the functional connectivity that developed during the trials with external stimulation and the values were intermediate between PRE/EARLY-learning and LATE-learning phase.

Table 2 shows the relative count of phase-coupling in the frequency bands of interest and the values of indexes $I R F$ and $R F R$ for all recording periods in controls and patients suffering primary insomnia before and after treatment [14]. The frequency ranges of the bands refer to those generally used for human studies and are different from those used for studying the Ubinet activity. However, there is a linear correspondence between the two sets of frequency bands. The 
general pattern was a high level of high frequency coupling in the group of patients before treatment. The main effect of the treatment was to reduce highfrequency coupling and shift phase-coupling towards low frequencies, somehow with a significant increase of low frequency coupling compared to the controls. The treatment significantly increased the phase-coupling in the low frequency band during all other intervals, either re-establishing a level close to the controls or even beyond that level, as observed during the REM sleep phases.

Table 1. Percentage of phase-coupled frequencies in each frequency bands of interest for the stimulus Type A and B within neural network development stages. IRF: index of resonant frequencies. RFR: raw frequency ratio.

\begin{tabular}{|c|c|c|c|c|c|}
\hline \multirow{2}{*}{$\begin{array}{c}\text { Learning } \\
\text { Phase }\end{array}$} & \multicolumn{3}{|c|}{ Percentage of phase-coupled frequencies } & \multicolumn{2}{|c|}{ Indexes } \\
\hline & LF: ] 1-24] Hz & ] $24-60] H z$ & HF: ]60-84] Hz & $I R F$ & $R F R$ \\
\hline \multicolumn{6}{|c|}{ Stimulus Type A } \\
\hline PRE & 27 & 53 & 20 & 43 & 1.34 \\
\hline EARLY & 20 & 50 & 30 & 60 & 0.66 \\
\hline LATE & 38 & 56 & 6 & 13 & 6.67 \\
\hline POST & 38 & 49 & 13 & 26 & 2.83 \\
\hline \multicolumn{6}{|c|}{ Stimulus Type B } \\
\hline PRE & 20 & 48 & 32 & 62 & 0.62 \\
\hline EARLY & 21 & 47 & 31 & 60 & 0.68 \\
\hline LATE & 49 & 43 & 8 & 14 & 6.00 \\
\hline POST & 44 & 38 & 18 & 29 & 2.43 \\
\hline
\end{tabular}

Table 2. Percentage of phase-coupled frequencies in each frequency bands of interest for the the control group and for the group of patients before and after treatment. $R E M$ : rapid eye movement sleep. NREM: rapid eye movement sleep.

\begin{tabular}{lllllr}
\hline \hline \multirow{2}{*}{ Subject Group } & \multicolumn{2}{c}{ Percentage of phase-coupled frequencies } & \multicolumn{3}{c}{ Indexes } \\
& LF: ] 1-13] Hz & ]13-33] Hz & HF: ]33-48] Hz & IRF & $R F R$ \\
\hline \hline \multicolumn{1}{c}{ Eyes Closed } & & & & & \\
Control & 12 & 74 & 14 & 54 & 1.17 \\
Patient before & 2 & 77 & 21 & 91 & 10.50 \\
after treatment & 8 & 88 & 4 & 33 & 0.50 \\
\hline NREM & & & & & \\
Control & 57 & 30 & 13 & 33 & 0.23 \\
Patient before & 27 & 60 & 13 & 2 & 0.48 \\
after treatment & 42 & 57 & 1 & & \\
\hline REM & & & & 56 & 1.25 \\
Control & 4 & 90 & 5 & 75 & 3.00 \\
Patient before & 4 & 85 & 12 & 10 & 0.11 \\
after treatment & 19 & 79 & 2 & & \\
\hline \hline
\end{tabular}




\section{Discussion}

This paper described the implementation of a neuronal system simulator on a hybrid scalable multi-agent hardware platform based on the Ubidules framework [9] and its application to the study of information processing in hierarchically organized neural networks circuits. We have explored one simple Ubinet network circuit characterized by a sensory network processing the external input that projects to a hierarchically organized multilayered (in our case formed by only two layers) recurrent network of processing areas which eventually project on a motor network that generates an activity keen to be encoded into actuators. The experimental approach to the Ubinet activity by recording the EChG was aimed to assess the effect of a repeated stimulation on the functional connectivity established between the Ubidules. Our PRE-learning stage could represent a control situation driven exclusively by the background activity of the subject's brain. The subject is naive to the coming stimulus so that a learning process can occur. During the EARLY-learning stage the repetition of the stimuli at regular intervals might initiate an unsupervised recognition process that eventually shaped the functional connectivity of feature detecting cell assemblies after selective synaptic and cell pruning.

The third order spectral analysis of EChG and EEG allows to determine the frequency range of quadratic phase coupling (resonant frequency) across cortical areas $[4,5]$. According to the usual interpretation based on standing waves theory, high resonant frequencies mean that information processing is transmitted at short distance (i.e., the distance between two nodes of the wave). A coupling that occurs at high frequencies may be interpreted as a sign of focal cortical interactions. Conversely, a coupling at low frequencies suggests an increased cross-areal involvement in neural processing.

A remarkable result is the finding that in the Ubinet simulations the LATElearning stages were characterized by $I R F \approx 14$ compared with $P R E$ - and $E A R L Y$-learning stages $(I R F \approx 43-62)$. In the study with human Subjects we observed that controls and patients after treatment were characterized, during all sleep phases by values of $I R F$ lower than insomniac patients before treatment. It is also worth reporting that the only condition that let appear a difference of resonant frequencies in the range ]13-33] $\mathrm{Hz}$ was during NREM sleep irrespective of the treatment. This last result suggests that despite an overall shift of resonant frequencies towards recovery, focal cortical interactions tended to persist in patients during NREM sleep periods. Both an appropriate stimulation of the Ubinet and the cognitive brain therapy appear to modify the ratio of resonant frequencies provoking a shift of the indexes towards low frequencies at all brain states. Our findings suggest that new tools provided by modular and scalable neural network simulators offer new opportunities to neurophysiologists and clinicians to test hypotheses based on the analysis of neural signals at mesoscopic levels.

Acknowledgments. The authors ackowledge the support by the European Union FP6 grant \#034632 (PERPLEXUs) and the contributions of J. Iglesias, 
Victor Contreras-Lámus for the simulations of O. Brousse, Th. Gil, G. Sassatelli, F. Grize for the JADE integration and of K. Espa-Cervena for the analysis of the clinical data.

\section{References}

1. Nunez, P.L., Srinivasan, R.: Electric Fields of the Brain. Oxford University Press, New York, NY, USA (2006)

2. Brillinger, D.R.: An introduction to polyspectra. Ann. Math. Stat. 36 (1965) 1351-1374

3. Dumermuth, G., Huber, P.J., Kleiner, B., Gasser, T.: Analysis of the interrelations between frequency bands of the EEG by means of the bispectrum. a preliminary study. Electroencephalogr. Clin. Neurophysiol. 31 (1971) 137-148

4. Villa, A.E.P., Tetko, I.V., Dutoit, P., De Ribaupierre, Y., De Ribaupierre, F.: Corticofugal modulation of functional connectivity within the auditory thalamus of rat. J. Neurosci. Meth. 86 (1999) 161-178

5. Villa, A.E.P., Tetko, I.V., Dutoit, P., Vantini, G.: Non-linear cortico-cortical interactions modulated by cholinergic afferences from the rat basal forebrain. BioSystems $\mathbf{5 8}$ (2000) 219-228

6. Iglesias, J., Villa, A.E.P.: Effect of stimulus-driven pruning on the detection of spatiotemporal patterns of activity in large neural networks. BioSystems 89 (2007) 287-293

7. Iglesias, J., Villa, A.E.P.: Emergence of preferred firing sequences in large spiking neural networks during simulated neuronal development. Int. J. Neural Syst. 18(4) (2008) 267-277

8. Shaposhnyk, V.V., Dutoit, P., Contreras-Lámus, V., Perrig, S., Villa, A.E.P.: A framework for simulation and analysis of dynamically organized distributed neural networks. Lecture Notes in Computer Science 5768 (2009) 277-286

9. Sanchez, E., Perez-Uribe, A., Upegui, A., Thoma, Y., Moreno, J.M., Villa, A., Volken, H., Napieralski, A., Sassatelli, G., Lavarec, E.: Perplexus: Pervasive computing framework for modeling complex virtually-unbounded systems. In: AHS '07: Proceedings of the Second NASA/ESA Conference on Adaptive Hardware and Systems, Washington, DC, USA, IEEE Computer Society (2007) 587-591

10. Bellifemine, F.L., Caire, G., Greenwood, D.: Developing Multi-Agent Systems With Jade. Wiley, Wiltshire, Great Britain (2007)

11. Brousse, O., Guillot, J., Sassatelli, G., Gil, T., Robert, M., Moreno, J.M., Villa, A., Sanchez, E.: A bio-inspired agent framework for hardware accelerated distributed pervasive applications. In: 2009 NASA/ESA Conference on Adaptive Hardware and Systems, Washington, DC, USA, IEEE Computer Society (2009) 415-422

12. Fipa, D.T.: Fipa communicative act library specification (2001)

13. Iglesias, J., Eriksson, J., Grize, F., Tomassini, M., Villa, A.E.: Dynamics of pruning in simulated large-scale spiking neural networks. BioSystems 79(1) (2005) 11-20

14. Perrig, S., Dutoit, P., Espa-Cervena, K., Shaposhnyk, V., Pelletier, L., Berger, F., Villa, A.E.P.: Changes in quadratic phase coupling of eeg signals during wake and sleep in two chronic insomnia patients, before and after cognitive behavioral therapy. Frontiers in Artificial Intelligence and Applications 204 (2009) 217-228 